\title{
DYNAMIC WHEEL-RAIL FORCES ON MISMATCHED JOINTS WITH RAMPS
}

\author{
Brian Marquis \\ Robert Greif \\ Volpe National Transportation Systems Center \\ US Department of Transportation \\ Cambridge, MA, USA
}

\begin{abstract}
The discontinuity between rail ends at a joint creates dynamic wheel-rail forces (i.e. high impact forces and wheel unloading) that can result in a range of problems including wear, deterioration, and early failure of the track structure, its components, and passing equipment. The response and magnitude of the dynamic wheel-rail forces generated at joints depend upon the form of the discontinuity (e.g. battered rail ends, ramps, gaps, mismatches, etc.) and the support condition. Joints with battered rail ends, which result from degradation due to repeated impact loading, have been extensively analyzed using closed form expressions developed by Jenkins [1] to estimate P1 and P2 impact forces. While appropriate for analyzing joints with battered rail ends, P1 and P2 forces are not directly applicable to other forms of discontinuity at joints such as mismatches in which the rail ends are offset vertically when installed.

Under certain circumstances, railroads are introducing ramps (by grinding or welding) to reduce the mismatch discontinuity and produce a smoother transition in order to mitigate these dynamic wheel-rail forces. In this paper, analyses are conducted to estimate dynamic wheel-rail forces at joints having ramps and mismatches of various sizes using simplified models along with detailed NUCARS models for comparative purposes. The Federal Railroad Administration (FRA) Track Safety Standards (49 CFR Part213) [2] limit the maximum mismatch at joints by Track Class in order to minimize the impact forces which deteriorate the track structure, its components, and equipment, and may ultimately lead to derailment. Parametric studies are conducted to examine the effects of ramp length, direction of travel, mismatch height, and equipment speed (track class). Plots of primary shock-response-spectrum (maximum impact force on the ramp), residual shock-response-spectrum (maximum impact force after the ramp), and minimum wheel force (i.e. wheel unloading) are developed to provide guidelines on ramp length (H-rule) in order to control the maximum force by track class.
\end{abstract}

\section{INTRODUCTION}

Figure 1 shows an example of a mismatched joint created in the field by mating two rails of different heights - namely, a new rail section on the right with a worn rail section on the left. In order to reduce dynamic wheel-rail forces from vehicles traversing such joints, railroads are introducing ramps (by grinding or welding) to reduce the mismatch discontinuity and produce a smoother transition. In this example, a ramp was ground on the end of the taller rail (new rail) to provide a transition between the two vertically offset running surfaces. This paper examines through modeling the effect of ramp geometry (ramp length and ramp mismatch) on reducing the dynamic vertical forces generated due to the interaction between the vehicle and track system. Combinations of ramp lengths and offset heights are investigated for a range of speeds.

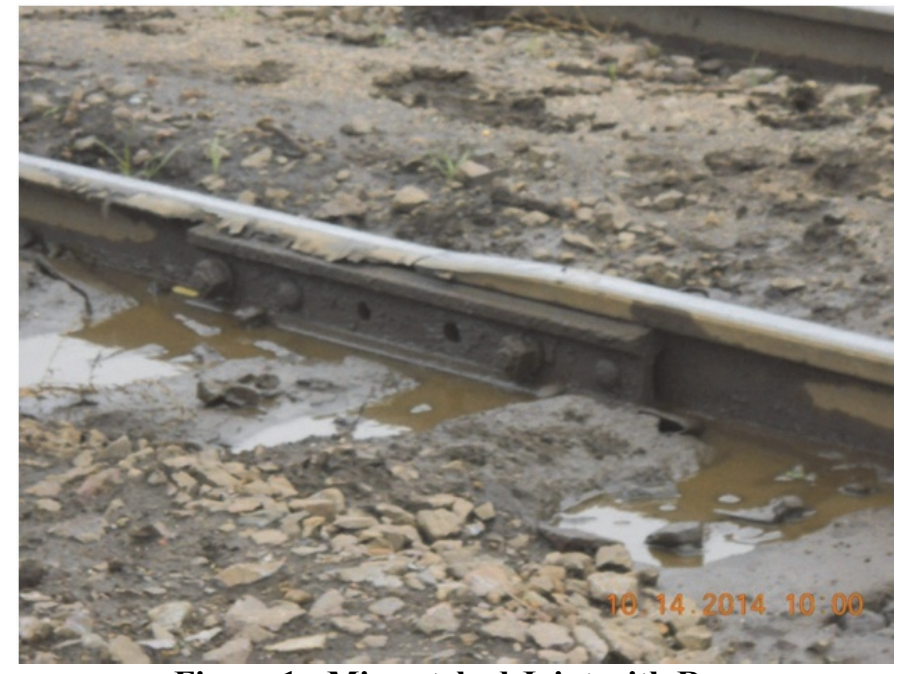

Figure 1: Mismatched Joint with Ramp 


\section{JOINT FORCES}

Figure 2 shows an idealized geometrical representation of a mismatched joint of vertical offset $\mathrm{H}$, with and without the application of a ramp of length $\mathrm{D}$ to minimize wheel-rail forces. The response of the vehicle to a ramp perturbation is driven by two separate input excitations, one from a discontinuity coming on the ramp and one from a discontinuity coming off the ramp. As a result of the spacing of the two discontinuities, the maximum vehicle responses (forces, displacements, and accelerations) may occur on the ramp or after coming off the ramp depending upon the time to traverse the ramp compared to the suspension characteristics of the vehicle.
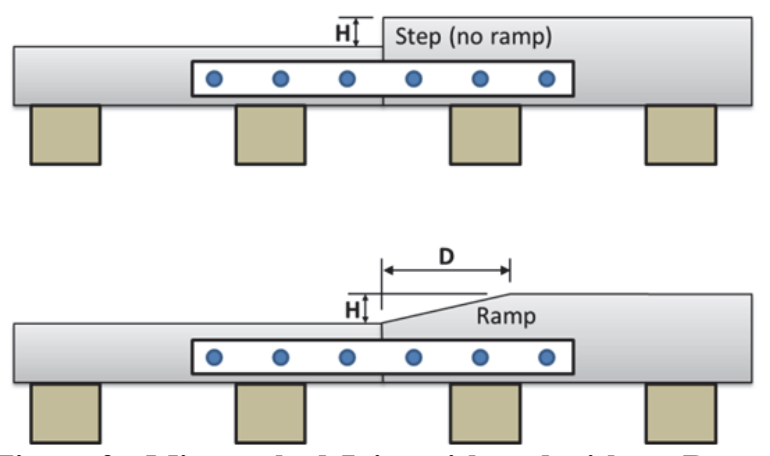

Figure 2: Mismatched Joint with and without Ramp

The dynamic motion of a wheel going over a small abrupt vertical change (such as a step or ramp) is influenced by two geometric features. First, the contact point between the wheel and rail shifts suddenly due to the geometric interference between the wheel and the lower running surface-rise transition. During this sudden shift two points of contact exist momentarily. As ramp length $\mathrm{D}$ increases, the vertical offset between the two contacts decreases for a fixed $\mathrm{H}$, as shown in Figure 3. As this offset between the two contacts decreases, the higher frequency wheel-rail impact response (which is part of the primary response, i.e. while on the ramp) becomes less pronounced at the lower running surface-rise transition.

Second, the upper running surface-rise transition acts as a pivot point about which the wheel rotates. Consequently, the motion of the center of the wheel is an arc of radius $\mathrm{R}$ resulting in a difference between the actual running surface and the input excitation to the primary suspension of the vehicle (shown as solid and dashed lines respectively in Figure 3). As the ramp length $\mathrm{D}$ increases, the arc length (which is part of the residual response, i.e. after coming off the ramp) decreases. As the arc length decreases, the motion more closely approaches that of a point mass traversing a ramp.
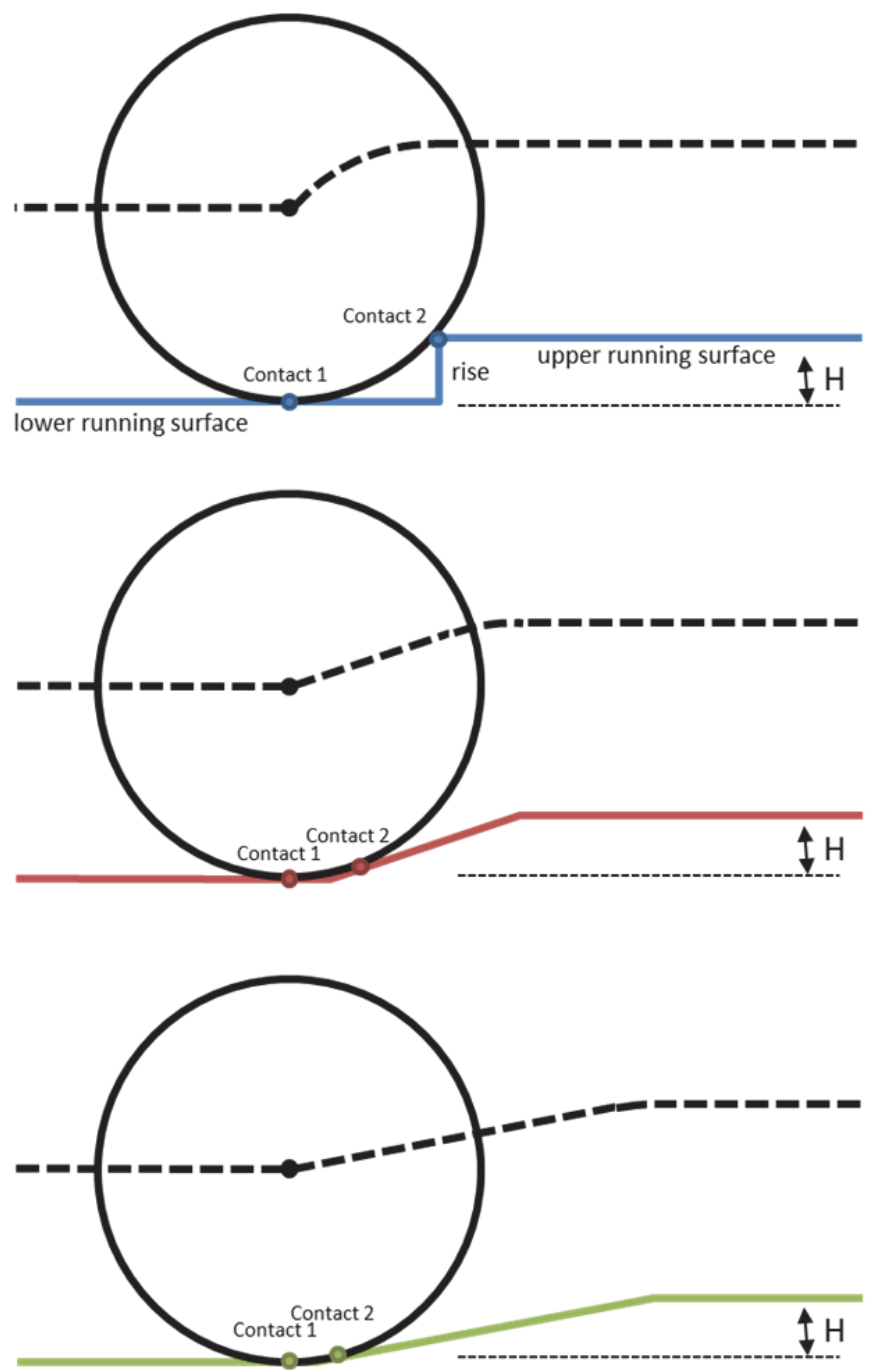

Figure 3: Wheel Path for Various Ramp (Rise) Lengths

Thus, in order to estimate the dynamic response of a vehicle to a mismatched rail, an analysis can be conducted using a single degree of freedom (SDOF) spring-mass system representation of a vehicle traveling with velocity $\mathrm{V}$ over a track with a ramp discontinuity shown in Figure 2. For this system, closed form solutions are available [3]. The carbody displacement $y(t)$ while on the ramp is

$$
y(t)=H\left(\frac{t}{t_{1}}-\frac{\sin \omega_{n} t}{\omega_{n} t_{1}}\right) \quad t<t_{1}
$$

where $\omega_{n}$ is the natural frequency of the vehicle, and $t_{1}$ (the total time spent on the ramp) is equal to $\mathrm{D} / \mathrm{V}$. Correspondingly, the estimated dynamic vertical force $F(t)$ while on the ramp is

$$
F(t)=k H\left(-\frac{\sin \omega_{n} t}{\omega_{n} t_{1}}\right) \quad t<t_{1}
$$


In a similar manner, the dynamic vertical force $F(t)$ along the track after coming off the ramp is

$$
F(t)=k H\left(-\frac{\sin \omega_{n} t}{\omega_{n} t_{1}}+\frac{\sin \omega_{n}\left(t-t_{1}\right)}{\omega_{n} t_{1}}\right) \quad t>t_{1}
$$

Combining equations 2 and 3 provides a closed form estimate for the undamped response for the dynamic vertical force of a railcar to a ramp discontinuity in the rail top profile. They show that the value of the dynamic force is related to the ramp duration time $t_{1}$ and the suspension characteristics of the vehicle $\tau$ (i.e. system period), where $\tau=2 \pi / \omega_{n}$ and $\omega_{n}$ depends on the suspended mass and stiffness of the vehicle. As a result, the dynamic vertical force for a ramp discontinuity is insensitive to the unsprung mass provided wheel separation does not occur and high-frequency wheel-rail impact is less prominent in the response. For other forms of joint discontinuities in which high frequency wheel-rail impacts are important, such as dipped rail joints, the unsprung mass can have a significant effect, as shown by Jenkins.

While the SDOF spring-mass system provides insight for interpreting the response and understanding the significant parameters affecting it, a detailed NUCARS model is also used to simulate the vertical motion of an actual railroad carbody moving, with velocity $\mathrm{V}$, over the rails containing the track discontinuity. These models are used to predict the time history of accelerations, displacements, and wheel-rail forces associated with a vehicle transitioning over the ramp to a new running height. The maximum value of these forces and their location on the rail are examined to evaluate safety while traversing different size ramp mismatches. By controlling the size of amplitude and length of the ramp, wheel-rail forces can be reduced to slow damage to the track structure and the rolling stock.

\section{RESULTS OF IDEALIZED MODEL}

For understanding the bounce behavior of a representative vehicle, Figure 4 shows the normalized SDOF carbody displacement response from motion traversing the ramp discontinuity. The horizontal axis represents time $t$ or distance $\mathrm{x}$ along the track normalized by the ramp rise time $\mathrm{t} 1$, or the ramp length $\mathrm{D}$, respectively. The response of the vehicle to the initial discontinuity when it comes on the ramp is the primary response, while the response to the second discontinuity when the vehicle comes off the ramp is the residual response. Each line represents the response for a specific ratio $\mathrm{T}$ which is equal to ramp time $t_{1}$ (seconds/ramp) divided by vehicle period $\tau$ (seconds/cycle). The ratio $\mathrm{T}$ can be interpreted as the number of cycles of vehicle response on the ramp, i.e. cycles/ramp. For $\mathrm{T}=1.0$, the ramp time and system period exactly match producing 1 cycle per ramp and a non-oscillatory residual response (i.e. in this case the vehicle second input excitation corresponding to the end of the ramp is coincident with the end of the vehicle's first oscillation cycle which has the effect of canceling the primary response and producing a non-oscillatory residual response). A similar residual response occurs for all integer values of $\mathrm{T}$ (so called nodes) as will be discussed further later. The results for non-integer values of $\mathrm{T}$ will depend on the phasing of the second input excitation (corresponding to the end of the ramp) to the cyclic motion of the primary response. The results for $\mathrm{T}=0.9$ and 0.5 show that decreasing $T$ below 1 is equivalent to shortening the ramp rise time $t_{1}$ (or ramp length for a fixed velocity), which produces a higher response that eventually will approach the maximum displacement response (step response) of 2 for very short rise times.

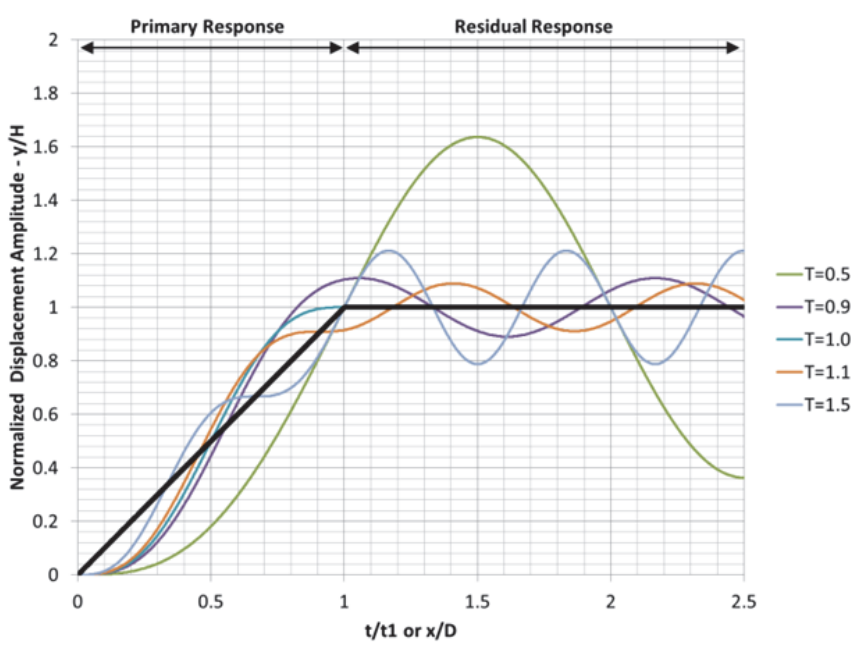

Figure 4: Normalized Vertical Displacement Response

Figure 5 shows the dynamic vertical force corresponding to the same representative "frequency" ratios $\mathrm{T}$ shown above in Figure 4. This dynamic force calculation is related to the difference between the carbody displacement of Figure 4 and the position of the wheel on the ramp or upper running surface.

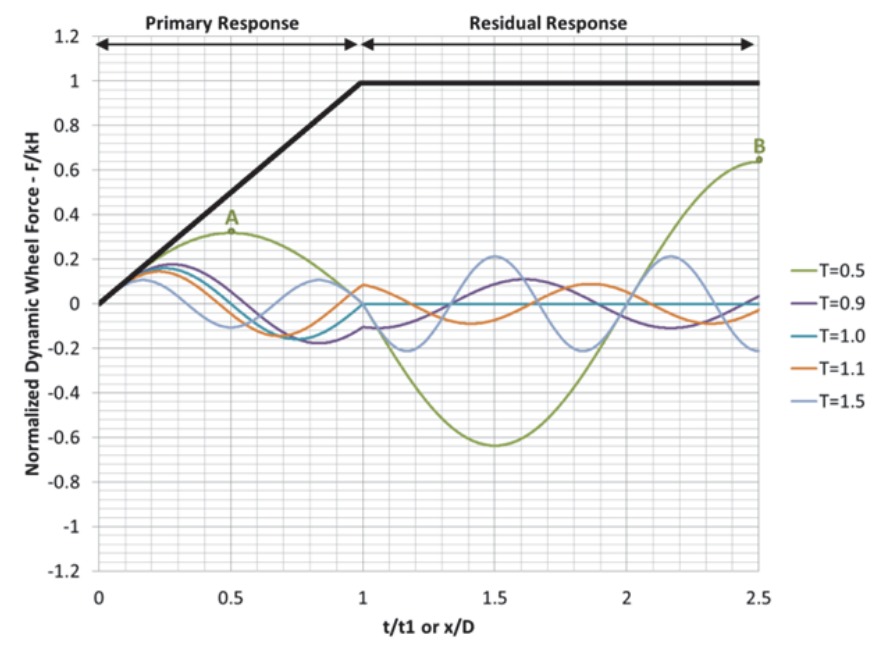

Figure 5: Normalized Dynamic Vertical Force Response 
The total wheel vertical force is the sum of the dynamic vertical force plus the static vertical force. For $\mathrm{T}=0.9,1.0$, and 1.1, the maximum dynamic vertical force occurs during the primary response. On the other hand, for $\mathrm{T}=0.5$ and $\mathrm{T}=1.5$ the maximum dynamic vertical force occurs during the residual response (for example, $\mathrm{B}$ is greater than $\mathrm{A}$ for the value of $\mathrm{T}=$ $0.5)$. These trends occur because the maximum force depends not only on the displacement of the carbody but also on the displacement of the wheel on the track as shown in Figure 3. As with the displacement response above, decreasing $\mathrm{T}$ is equivalent to shortening the ramp rise time $t_{1}$ (or ramp length for a fixed velocity), which produces a higher response that eventually will approach the maximum dynamic force response (step response) of 1 for very short rise times.

Figure 6 presents plots of primary and residual dynamic vertical force shock-response spectrum (SRS). This is obtained by first calculating the dynamic vertical force for a wide range of $\mathrm{T}$ (similar to those shown in Figure 5) and identifying the maximum primary and residual responses for each value of $T$ (for example the $\mathrm{A}$ and $\mathrm{B}$ are the maximum primary and residual responses for a value of $\mathrm{T}=0.5$ in Figure 5). These curves show that as $\mathrm{T}$ increases from zero, which is equivalent to starting with a step input excitation and increasing the ramp length or rise time, the overall maximum response occurs during the residual response range (namely $\mathrm{B}>\mathrm{A}$ ) except in the vicinity of integer $T$ values where the overall maximum occurs during the primary response. This phenomenon of switching the location of the overall maximum from residual to primary response and back again (i.e. the maximum is on the ramp or after the ramp) occurs when the end of an oscillation cycle of the vehicle (system) is equal to or near the end of the ramp.

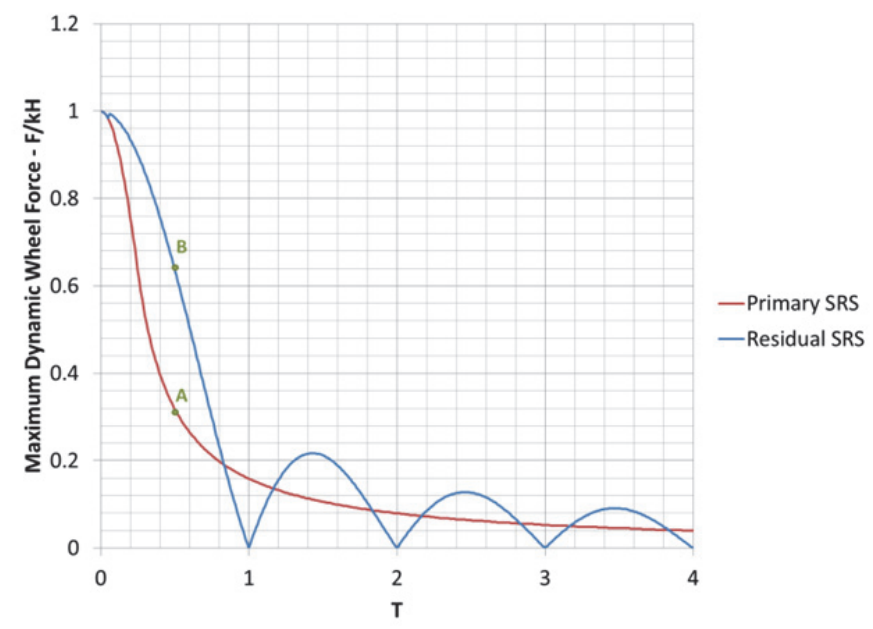

Figure 6: Dynamic Vertical Force Spectrum

\section{RESULTS OF DETAILED MODEL}

Table 1 lists the maximum vertical rail-end mismatch of rails at joints for each class of track defined in 49CFR $\S$ 213.115 of the FRA Track Safety Standards [2].
Table 1: FRA Tread Rail-End Mismatch (49 CFR 213.115)

\begin{tabular}{|l|c|}
\hline & $\begin{array}{c}\text { Mismatch } \\
\text { (inches) }\end{array}$ \\
\hline Class 1 (10 mph) & 0.25 \\
\hline Class 2 (25 mph) & 0.25 \\
\hline Class 3 (40 mph) & 0.1875 \\
\hline Class 4 (60 mph) & 0.125 \\
\hline Class 5 (80 mph) & 0.125 \\
\hline
\end{tabular}

Figure 7 shows the vertical wheel force response of a 286 kip loaded hopper car predicted using the maximum mismatch and speed permitted for each track class. The hopper car response is predicted with NUCARS using a detailed full vehicle model having many degrees of freedom. The maximum wheel force occurs at the instant of step excitation followed by a transient oscillatory response, similar to that seen in the SDOF modeling for a pure step. However, note that the SDOF model leading to Figure 4 is for a ramped track, so that the maximum wheel force occurs either along the ramp or on the upper running surface

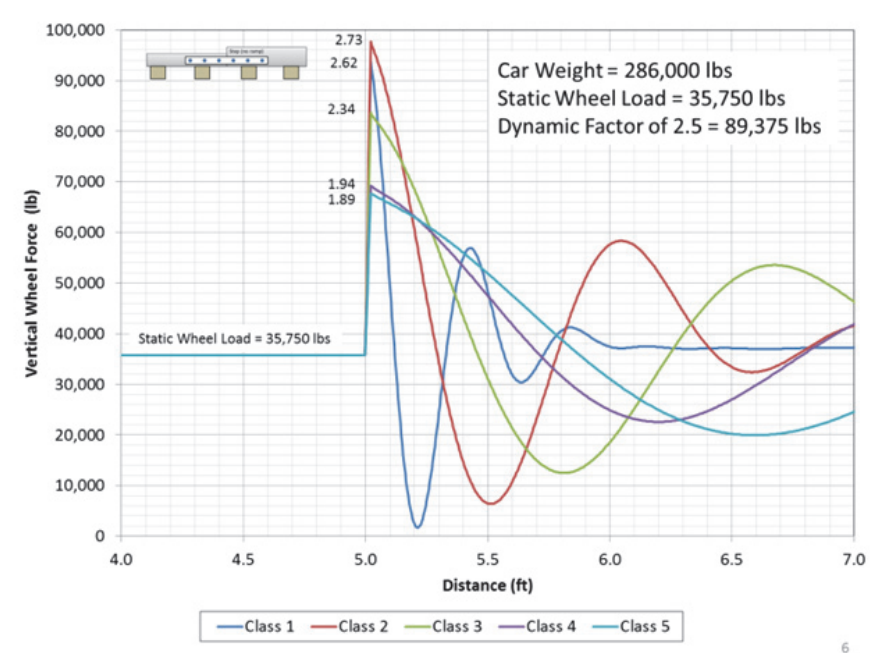

Figure 7: Vertical Wheel Force for each Track Class

Figure 8 summarizes the maximum mismatch $\mathrm{P}$ in blue and the maximum vertical wheel force $\mathrm{Q}$ in red for each Track Class. For convenience, the vertical force is expressed as a dynamic ratio to the static wheel load of 35,750 lbs (for example a dynamic ratio of 2.5 corresponds to a vertical load of $89,375 \mathrm{lbs}$. The Class 1 and Class 2 NUCARS results are for the same tread mismatch input but different speeds $(10-25$ mph). Similarly, the Class 4 and Class 5 NUCARS results have equal tread mismatch but different speeds $(60-80 \mathrm{mph})$. It is interesting to note that for an undamped SDOF model the Class 1 and 2 maximum force values are equal to each other, and a similar result occurs for the Class 4 and 5 maximum forces. However, for a SDOF model which includes damping, the equality of these respective maximum forces no longer holds and small differences can appear, like those shown in Figure 8. 


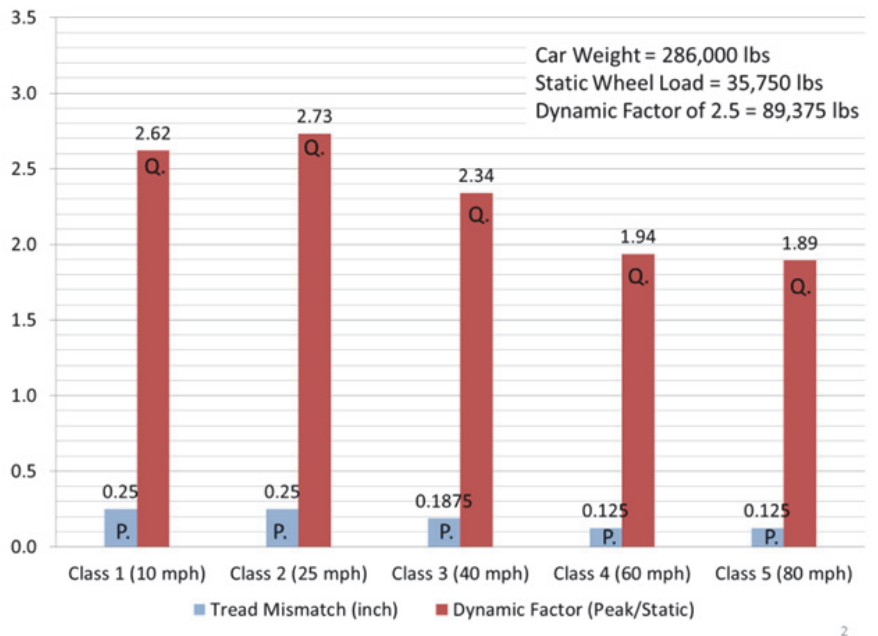

Figure 8: Summary of Maximum Vertical Wheel Force for each Track Class

Table 2 summarizes the Vehicle-Track Interaction (VTI) safety limits on wheel/rail forces in the FRA Track Safety Standards (49 CFR Part213) [2]. While a limit on minimum wheel load is included (single wheel vertical load ratio), FRA does not include a limit on the maximum allowable dynamic wheel force. Thus, for the purposes of establishing recommendations on ramp lengths, the results shown in Figure 8 are used in this paper to represent the maximum safe load for each Track Class.

Table 2: FRA VTI Safety Criteria (49 CFR 213.333)

\begin{tabular}{|c|c|c|c|}
\hline & $\begin{array}{c}\text { Safety } \\
\text { Concern }\end{array}$ & Parameter & Safety Limit \\
\hline \multirow{4}{*}{ 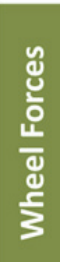 } & Wheel Unloading & $\begin{array}{l}\text { Single Wheel Vertical } \\
\text { Load Ratio }\end{array}$ & $\geq 0.15$ \\
\hline & $\begin{array}{l}\text { Wheel-climb } \\
\text { Derailment }\end{array}$ & Single Wheel L/V & $\leq \frac{\tan (\delta)-0.5}{1+0.5 \tan (\delta)}$ \\
\hline & Track Shift & Net Axle L/V & $\leq 0.4+\frac{5.0}{V a}$ \\
\hline & Rail Rollover & Truck-side L/V & $\leq 0.60$ \\
\hline
\end{tabular}

Figure 9 shows the maximum wheel force for combinations of tread mismatch and ramp length. The solid black line shows the maximum Class 4 dynamic vertical wheel force ratio of 1.94 for a maximum tread mismatch of 0.125 inches shown in Figure 8. The top blue line shows how the maximum dynamic vertical wheel force ratio increases with increasing tread mismatch for a step excitation (i.e. zero length ramp). Each of the colored lines below the top blue line show how the maximum dynamic vertical wheel force ratio decreases if a ramp of various lengths were introduced. The curves show that as ramp length increases (from 2 inches to 20 inches) the maximum dynamic vertical force ratio decreases for a given tread mismatch.

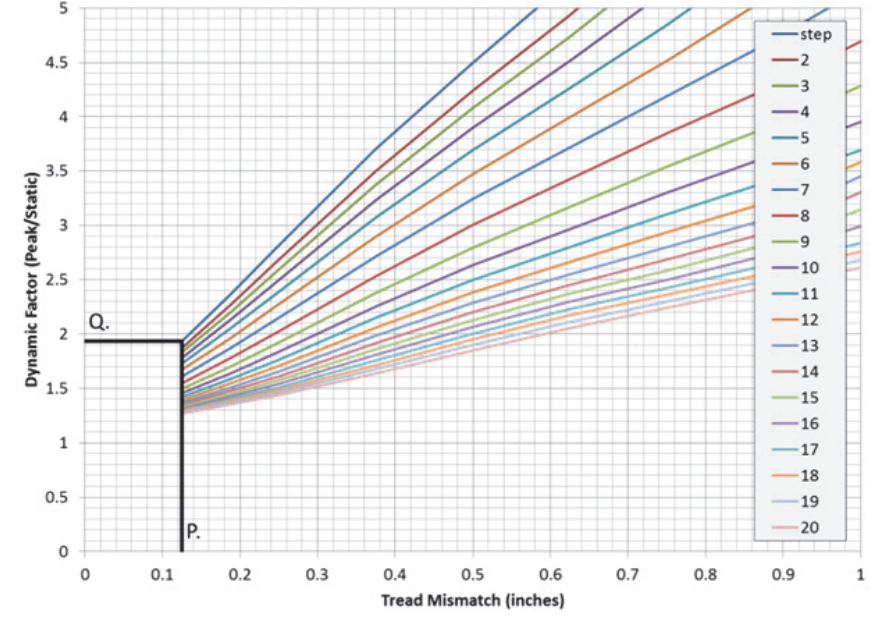

Figure 9: Track Class 4 Maximum Vertical Wheel Force for Varying Tread Mismatch and Ramp Lengths (inches)

Assuming the maximum dynamic force ratio noted in Figure 8 is the maximum allowable safe force (as mentioned previously), the results in Figure 9 can be divided into 2 zones - combinations of tread mismatch and ramp length producing unsafe forces and combinations producing safe forces, as shown as shaded and unshaded zones respectively in Figure 10.

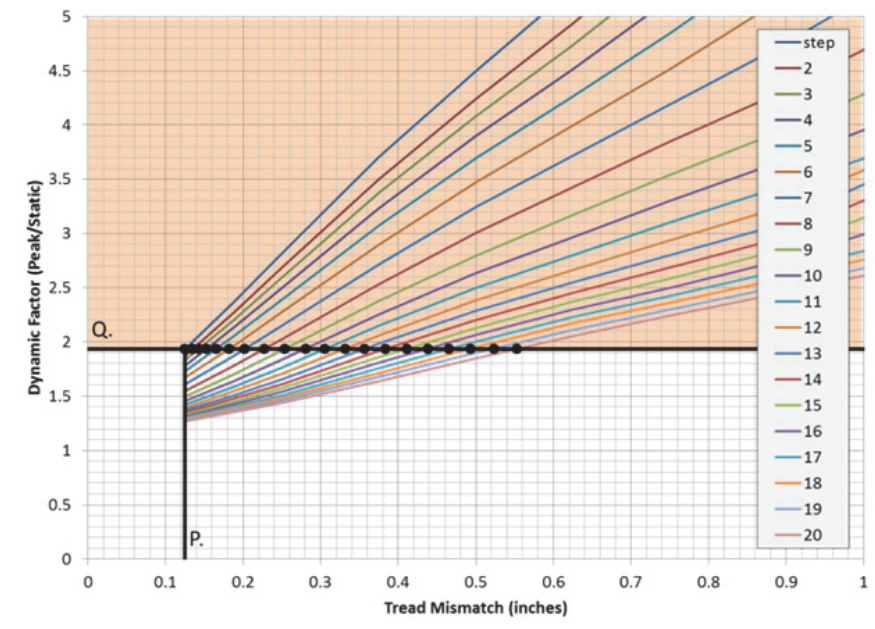

Figure 10: Track Class 4 Maximum Vertical Wheel Force for Varying Tread Mismatch and Ramp Lengths (inches) with Unsafe Zone Shaded

The intersection of each of the colored lines which correspond to ramps of different lengths and the "safe/unsafe" boundary (horizontal black line) is denoted by black dots. These points represent the combinations of tread mismatch and ramp length to produce a dynamic vertical wheel force ratio equal to 1.94. A cross plot of these points for this constant force is shown in Figure 11. Combinations of tread mismatch and ramp length above the line produce a lower dynamic vertical force ratio (safe) while combinations below produce a 


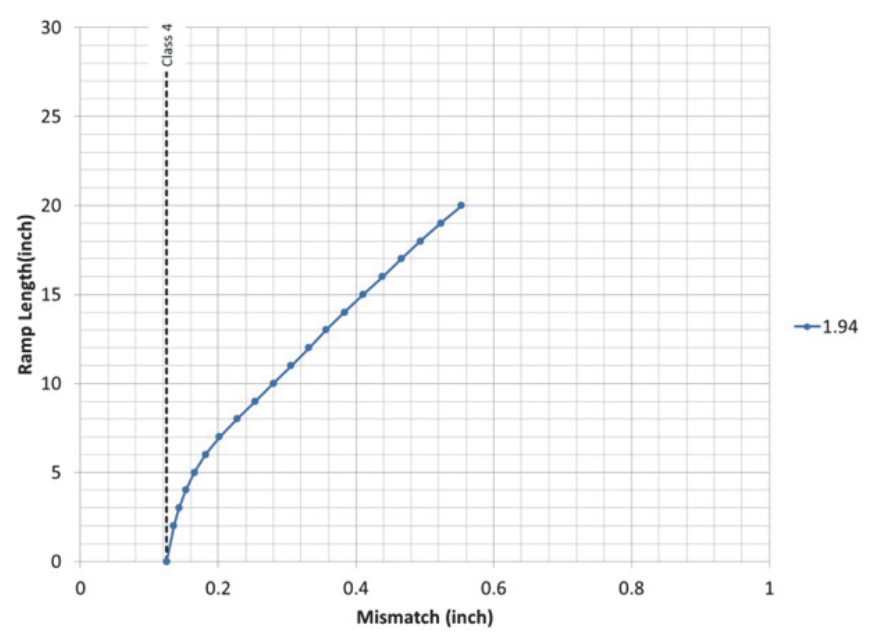

Figure 11: Combination of Tread Mismatch and Ramp Length to Produce Maximum Dynamic Vertical Wheel Force Ratio of 1.94 for Class 4

higher dynamic vertical wheel force ratio (unsafe).

The "safe/unsafe" boundary shown in Figure 11 can also be derived by inverting and scaling the Primary SRS results in Figure 6 as shown in Figure 12. The "safe/unsafe" boundaries in both models have an initial bend followed by a linear portion. In this case, because of the close agreement, the SDOF is convenient for interpreting the shape of the relationship obtained in the detailed model.

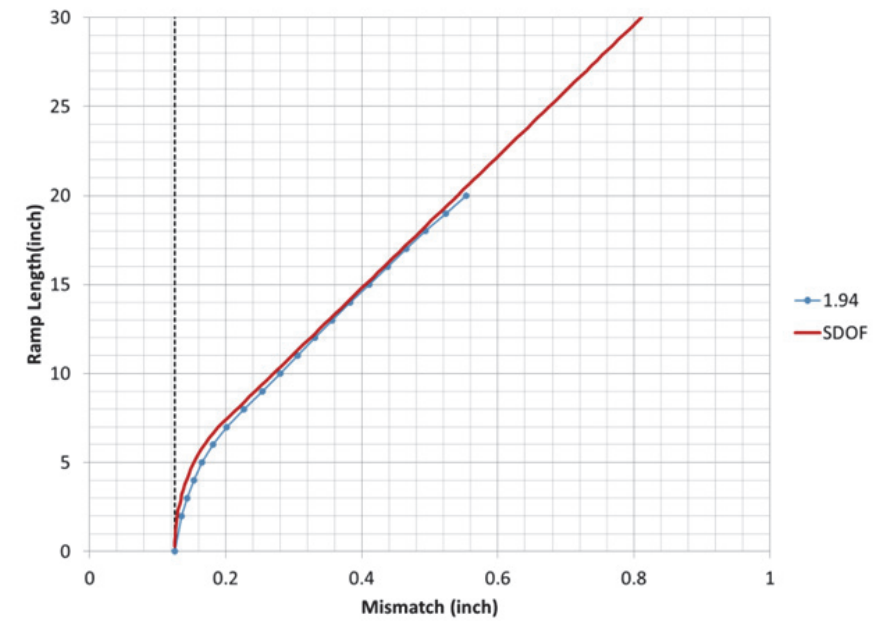

Figure 12: Combination of Tread Mismatch and Ramp Length to Produce Maximum Dynamic Vertical Wheel Force Ratio of 1.94 for Class 4

Figure 13 shows that the slope of the linear portion of the "safe/unsafe" boundary increases with increasing speed (holding all else in Figure 12 constant). The increasing slope is physically consistent given that long ramps will appear shorter from a vehicle perspective at higher speeds and will eventually look like a step (i.e. the "safe/unsafe" response will be a vertical line for infinite speed indicating all ramp lengths produce the same force for the same mismatch height everything looks like a step). This can also be seen when referring back to the results with the idealized model noting that an increase in speed is similar to a decrease in $t 1$, i.e. more like a step.

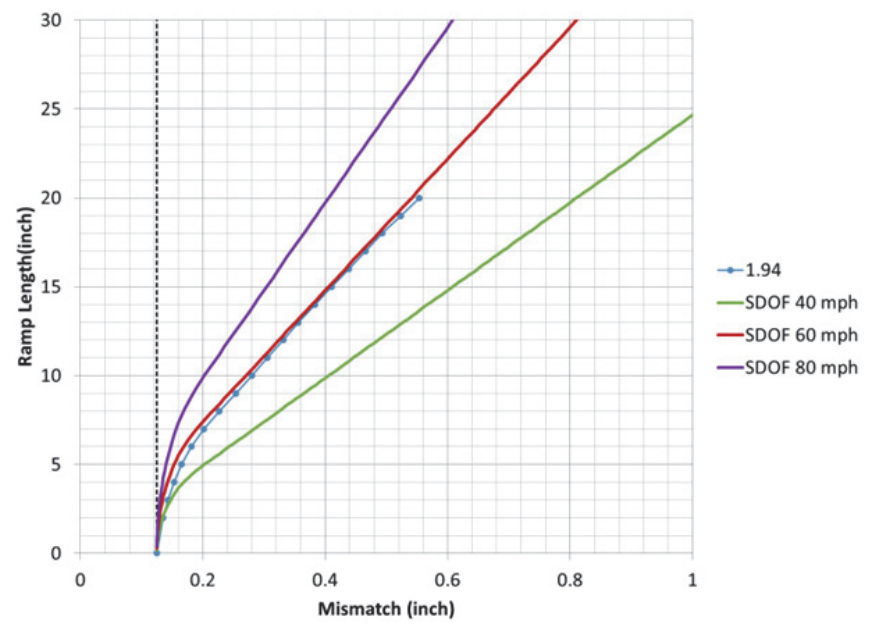

Figure 13: Combination of Tread Mismatch and Ramp Length to Produce Maximum Dynamic Vertical Wheel Force Ratio of 1.94 for Various Speeds

Similar "safe/unsafe" boundaries to those shown in Figure 11 have been generated using the detailed model for each of the Track Classes from 1 to 5 as shown in Figure 14.

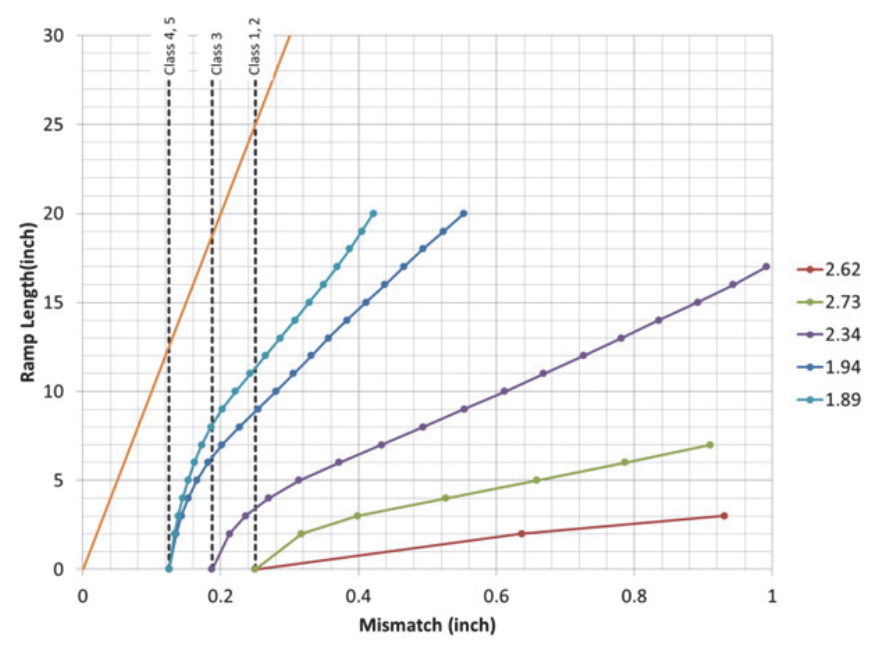

Figure 14: Combination of Tread Mismatch and Ramp Length to Produce Maximum Dynamic Vertical Wheel Force Ratio for each Track Class

Based on the results shown in Figure 14, and parametric results with the SDOF model, a ramp length D greater than or 
equal to 100 times the mismatch height is recommended as a conservative general rule of thumb to control the maximum force and provide a level of safety equal to or better than the current allowable limits on tread mismatch. This recommendation (referred to as the H-rule) is shown in gold in Figure 14. Such a ramp can be verified in the field using a straight edge as shown in Figure 15.

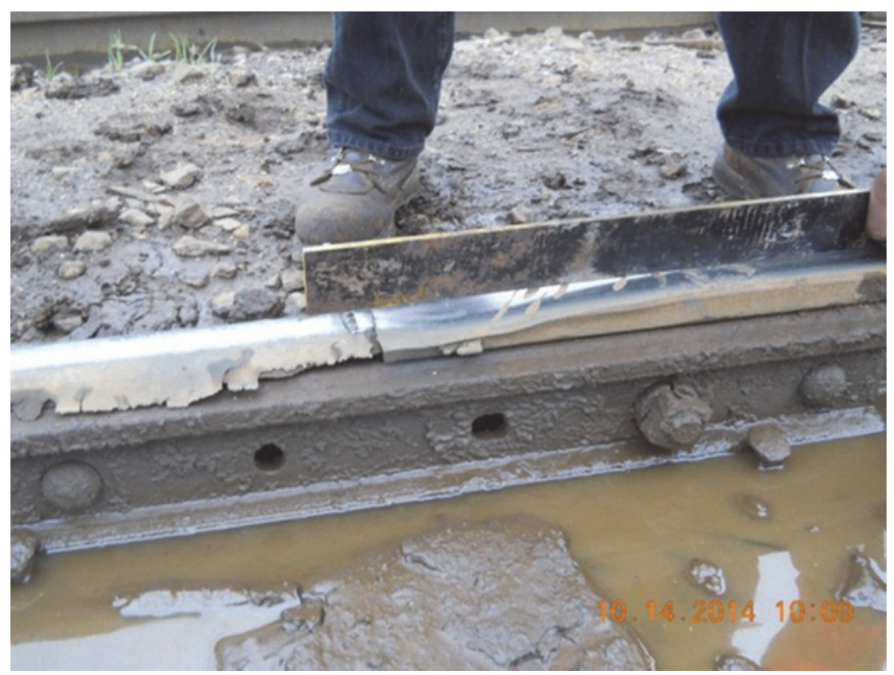

Figure 15: Field Measurement of the Mismatched Joint with Ramp Shown in Figure 1

\section{CONCLUDING REMARKS}

In this paper an analysis was done to investigate the effect of ramp discontinuities which are introduced (by grinding or welding), when a joint is installed between two rails vertically offset, in order to produce a smoother transition and reduce the dynamic vertical wheel-rail forces applied by passing vehicles. Amongst other things, these forces contribute to progressive deterioration of the track infrastructure (especially at the joint) and will likely become worse as this occurs. The dynamic vertical forces are calculated using vehicle models of varying complexity to understand the effects of ramp geometry including length and mismatch offset. Results are presented for maximum speeds and permissible vertical mismatches corresponding to each class of track. A general recommendation $(\mathrm{D} \geq 100 \mathrm{H}$ ) is established for ramp length $\mathrm{D}$ in terms of the mismatch height $\mathrm{H}$ needed to control the maximum force by track class and provide an equivalent or better level of safety. Future work is intended to examine the effect of other vehicle types, the effect of ramps on wheel unloading and measured wheel-rail forces to validate the responses shown in this paper.

\section{ACKNOWLEDGMENTS}

The work described in this paper was sponsored by the Office of Research and Development, Federal Railroad Administration (FRA), U.S. Department of Transportation.

\section{REFERENCES}

1. Jenkins,H.H.,et.al, "The Effect of Track and Vehicle Parameters on Wheel/Rail Vertical Dynamic Forces" , Railway Engineering Journal, 1974, pp 2-26.

2. U.S. Department of Transportation, Federal Railroad Administration, 49 CFR Parts 213.

3. Thomson, W.T. And Dahleh, M.D., "Theory of Vibration with Applications", Prentice - Hall, 1993. 